\title{
The nature of gamma ray blazar candidate PMN J1326-5256
}

Hayley Bignall*, Giuseppe Cimò

Joint Institute for VLBI in Europe

E-mail: bignallejive.nl, cimodjive.nl

\section{David Jauncey}

Australia Telescope National Facility, CSIRO

E-mail: David.Jauncey@csiro.au

\section{Cliff Senkbeil, Jim Lovell, Simon Ellingsen}

University of Tasmania

E-mail: cliffs@utas.edu.au, Jim.Lovell@utas.edu.au,

Simon.Ellingsen@utas.edu.au

\begin{abstract}
A comparison of AGN detected at gamma ray energies by EGRET with flat-spectrum radio sources observed in surveys for intraday variability reveals that a remarkably high fraction of EGRET blazars show significant interstellar scintillation at centimetre wavelengths. Scintillating AGN will therefore be targets of interest for GLAST, scheduled for launch in early 2008. We suggest that the variable, scintillating flat-spectrum radio source PMN J1326-5256 is associated with the unidentified EGRET source 3EG J1316-5244. We describe the properties of PMN J1326-5256 and present recent results of monitoring with the ATCA and Ceduna radio telescopes.
\end{abstract}

From planets to dark energy: the modern radio universe October 1-5 2007

University of Manchester, Manchester, UK

\footnotetext{
* Speaker.
} 


\section{Multiwavelength properties of PMN J1326-5256}

There is a strong connection between AGN exhibiting interstellar scintillation (ISS) and blazars that were detected at gamma ray energies with EGRET. Out of the 19 EGRET blazars observed in the MASIV $5 \mathrm{GHz}$ VLA Survey [1. 2], 17 showed significant intraday variability (IDV) in at least one epoch with rms fractional variations 1-6\%; in comparison, $56 \%$ of the entire compact, flatspectrum MASIV sample showed such IDV. The MASIV survey showed a strong Galactic latitude dependence of IDV, indicating a predominantly interstellar origin for IDV at $5 \mathrm{GHz}$.

The radio source PMN J1326-5256 is a little-studied object with very few references in the literature, and no optical identification prior to the present work. It was observed in the ATCA ${ }^{1}$ calibrator survey and discovered to be intraday variable (R.J. Sault 2001, private communication). The source has no large-scale structure, being completely unresolved with the ATCA and also the AT-LBA with a maximum resolution of 16 mas at $2.3 \mathrm{GHz}$. The most accurate $\mathrm{J} 2000$ coordinates available for PMN J1326-5256 are $13^{\mathrm{h}} 26^{\mathrm{m}} 49.23^{\mathrm{s}} \pm 0.02^{\mathrm{s}},-52^{\circ} 56^{\prime} 23.7^{\prime \prime} \pm 0.1^{\prime \prime}$, determined from ATCA data at $4.8 \mathrm{GHz}$. This position is coincident with sources in the USNO-A2 and 2MASS catalogues. We obtained an optical spectrum during AAT service observations on 5 June 2002. The spectrum is featureless across the observed range of $\sim 5000-9000 \AA$, with $\mathrm{S} / \mathrm{N} \sim 15$ in the unaveraged continuum. During the AAT observations it was noted that the object appeared much brighter than on archival UK Schmidt plates, indicating strong optical variability. Near-infrared colours from 2MASS ( $\mathrm{J}=14.67, \mathrm{H}=13.70, \mathrm{~K}=12.78$ ) match typical BL Lacertae objects. PMN J1326-5256 is a candidate BL Lac object, although further optical spectroscopy, particularly in the blue end of the spectrum, would be useful to confirm this identification.

3EG J1316-5244 is an unidentified EGRET source at an angular separation of $1.5^{\circ}$ from PMN J1326-5256. This offset is larger than the quoted error radius of $0.5^{\circ}$, however we note that 3EG 1316-5244 is flagged as having an irregular or not closed 95\% position likelihood contour, indicating possible large uncertainty in the EGRET source location [3]. We suggest a tentative association between 3EG J1316-5244 and PMN J1326-5256 based on multiwavelength properties.

\section{Scintillation monitoring}

An annual cycle in the timescale of interstellar scintillation (ISS) is expected from the changing velocity of the Earth relative to the scattering plasma. In principle, observations of ISS at different times of the year can be used to determine scattering screen parameters and microarcsecond-scale source structure. PMN J1326-5256 was included in an ATCA IDV monitoring programme, in which it was observed at 4.8 and $8.6 \mathrm{GHz}$ in 14 sessions of $\sim 2$ days or more, over a period of 2.5 years starting in early 2001, shortly after the discovery of its IDV. This source showed the largest amplitude IDV of the 21 IDV sources included in the ATCA monitoring programme, with a maximum modulation index (fractional rms variation) of $16 \%$ at both 4.8 and $8.6 \mathrm{GHz}$, although significant IDV was not observed in every epoch, with the 2-day modulation index being $\lesssim 1 \%$ at times. The MASIV Survey showed that episodic IDV is a common phenomenon amongst flatspectrum radio sources [2].

\footnotetext{
${ }^{1}$ The Australia Telescope Compact Array is part of the Australia Telescope which is funded by the Commonwealth of Australia for operation as a National Facility managed by CSIRO.
} 


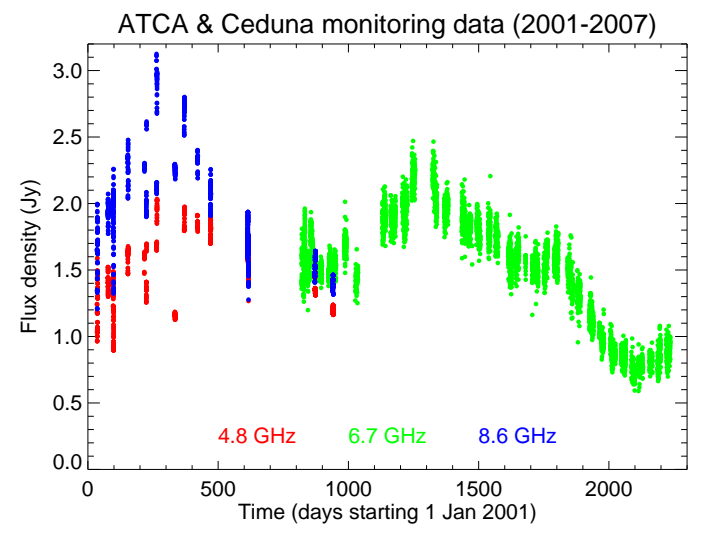

Figure 1: Six years of ATCA and Ceduna total flux density monitoring.

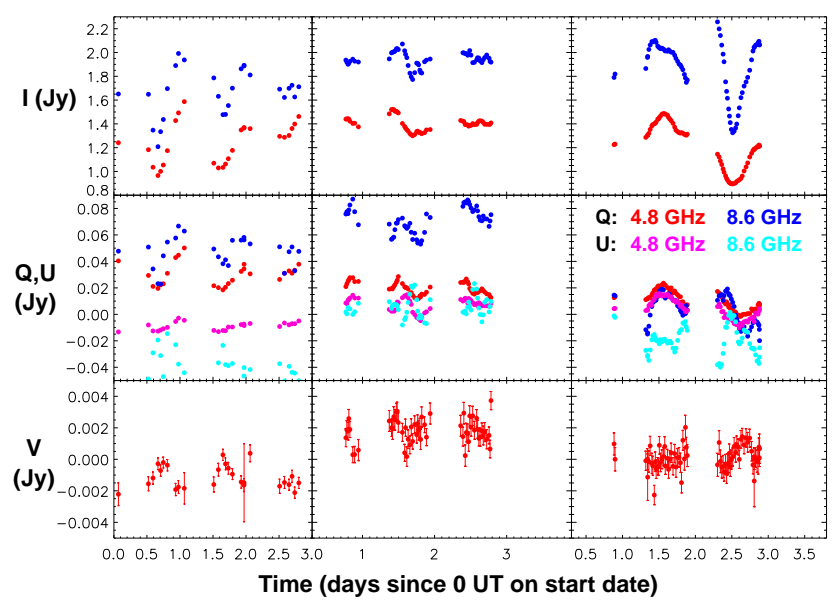

Figure 2: First three epochs of ATCA data, starting on 4 February, 17 March and 6 April, 2001, showing all four Stokes parameters plotted with 5-minute or scan averaging.

PMN J1326-5256 is also a target of the COSMIC project [ $₫$ ], using the University of Tasmania's 30-m radio telescope at Ceduna. This programme aims at dedicated monitoring of several IDV sources at 6.7 GHz. COSMIC observations of PMN J1326-5256 started in early 2003. Figure 1 shows ATCA and Ceduna monitoring data up to early 2007. Figure 2 shows all four Stokes parameters plotted for the first three epochs of ATCA data. Stokes V (circular polarization) shows a sign flip between the first two epochs which helps to constrain the origin of the circularly polarized radiation. The variations at 4.8 and $8.6 \mathrm{GHz}$ are strongly correlated, indicative of scintillation in the weak scattering regime, but at Galactic coordinates $l=308.3^{\circ}, b=9.6^{\circ}$, PMN J1326-5256 would be expected to undergo strong scattering at these frequencies. An additional nearby scattering "screen" may be responsible for the rapid IDV observed, since scattering material close to the observer has a lower transition frequency and causes more rapid variations than the same material at a larger distance.

There is some evidence of a slow-down in the ISS timescale around November from the first 
year of ATCA data and later Ceduna data, but no clear repeating annual cycle is seen. The IDV displays large changes in modulation index (fractional rms variation) at different epochs. Currently it is not clear whether changes in the IDV behaviour of PMN J1326-5256 are mostly due to intrinsic source changes or changes in the interstellar medium along the line-of-sight. Because of the low Galactic latitude of PMN J1326-5256, the distribution of scattering plasma along the line-of-sight to this source is likely to be complex. A superposition of multiple scattering "screens" could lead to multiple scintillation timescales, which are evident in the Ceduna monitoring data. If the edge of one such scattering region drifts in or out of the line-of-sight to PMN J1326-5256, this could result in changes in the ISS behaviour. From Figure 1, however, it is clear that sourceintrinsic changes occur on timescales of months to years. From the ATCA monitoring, the observed decrease in modulation index with time shows a moderate correlation $(\rho \sim 0.6)$ with steepening spectral index, suggesting a possible expansion, quenching of the ISS and subsequent fading of a compact component. We have investigated the radio spectral variability of PMN J1326-5256 using data between 1.4 and $96 \mathrm{GHz}$ from the ATCA calibrator database, observed irregularly between 2000 and 2007. Taking data from epochs close in time to estimate instantaneous spectra, there is evidence that during the period where the source displayed rapid IDV, the spectrum peaks above $22 \mathrm{GHz}$, indicating the presence of a strongly self-absorbed synchrotron component, while at later times during the "quiescent" phase observed at Ceduna, the turnover frequency dropped down below $10 \mathrm{GHz}$. Recently the spectrum has again started to become more inverted. Continued monitoring and more detailed analysis and modelling of the Ceduna light curves are therefore of interest to determine the origin of the observed changes in the scintillation of PMN J1326-5256.

\section{Summary}

The radio spectrum, extreme ISS and longer-term intrinsic radio variability of PMN J13265256 imply the presence of a high brightness temperature, highly beamed jet component. A first optical spectrum of the source, together with the photometric properties indicate that it is a typical low-frequency peaked (classical "radio-selected") BL Lac object. We suggest a tentative association with the unidentified EGRET source 3EG J1316-5244. GLAST will have the resolution and sensitivity to be able to confirm PMN J1326-5256 as a gamma ray source. Multiwavelength monitoring of sources such as PMN J1326-5256 in the GLAST era has the potential to make important contributions to our understanding of the physics of AGN jets and high energy emission mechanisms.

\section{References}

[1] J.E.J. Lovell et al., First Results from MASIV: The Microarcsecond Scintillation-Induced Variabilty Survey, AJ 126 (2003) 1699

[2] D.L. Jauncey et al., Statistics of the MASIV 5 GHz VLA Scintillation Survey, these proceedings

[3] R.C. Hartman et al., The Third Egret Catalog of High-Energy Gamma-Ray Sources, ApJS 123 (1999) 79

[4] P.M. McCulloch et al., COSMIC: Microarcsecond Resolution with a 30 Meter Radio Telescope, AJ 129 (2005) 2034 